\section{(6) OPEN ACCESS}

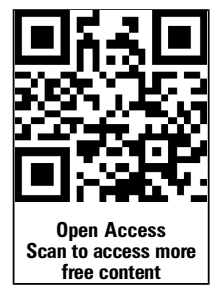

For numbered affiliations see end of article.

\section{Correspondence to} Dr Bart Nuttin, Department of Neurosurgery, UZ Leuven, KU Leuven, Herestraat 49, 3000 Leuven Belgium; bart.nuttin@ kuleuven.be

BN and HW contributed equally to this study.

From the World Society for Stereotactic and Functional Neurosurgery (WSSFN), the Working Group 'Deep Brain Stimulation in Psychiatry: Guidance for Responsible Research and Application', the European Society for Stereotactic and Functional Neurosurgery (ESSFN), the American Society for Stereotactic and Functional Neurosurgery (ASSFN), the Latin American Society for Stereotactic and Functiona Neurosurgery (SLANFE), the Asian- Australasian Society for Stereotactic and Functional Neurosurgery (AASSFN) and the World Psychiatric Association (WPA)

Received 25 September 2013 Revised 30 November 2013 Accepted 18 December 2013 Published Online First 20 January 2014

\title{
Consensus on guidelines for stereotactic neurosurgery for psychiatric disorders
}

Bart Nuttin, ${ }^{1,2,3,4}$ Hemmings $\mathrm{Wu}_{1}{ }^{1}$ Helen Mayberg, ${ }^{3,5}$ Marwan Hariz, ${ }^{4,6}$ Loes Gabriëls, ${ }^{4,7}$ Thorsten Galert, ${ }^{3,8}$ Reinhard Merkel, ${ }^{3,9}$ Cynthia Kubu, ${ }^{3,10}$ Osvaldo Vilela-Filho, ${ }^{4,11}$ Keith Matthews, ${ }^{4,12}$ Takaomi Taira, ${ }^{13}$ Andres M Lozano, ${ }^{14}$ Gastón Schechtmann, ${ }^{4,15}$ Paresh Doshi, ${ }^{4,16}$ Giovanni Broggi, ${ }^{4,17}$ Jean Régis, ${ }^{4,18}$ Ahmed Alkhani, ${ }^{4,19}$ Bomin Sun, ${ }^{4,20}$ Sam Eljamel, ${ }^{4,21}$ Michael Schulder, ${ }^{22}$ Michael Kaplitt, ${ }^{23}$ Emad Eskandar, ${ }^{24}$ Ali Rezai, ${ }^{25}$ Joachim K Krauss, ${ }^{26}$ Paulien Hilven, ${ }^{27}$ Rick Schuurman, ${ }^{28}$ Pedro Ruiz, ${ }^{29}$ Jin Woo Chang, ${ }^{4,30}$ Paul Cosyns, ${ }^{31}$ Nir Lipsman, ${ }^{4,32}$ Juergen Voges, ${ }^{4,33}$ Rees Cosgrove, ${ }^{4,34}$ Yongjie Li, ${ }^{35}$ Thomas Schlaepfer ${ }^{3,36}$

\section{ABSTRACT}

Background For patients with psychiatric illnesses remaining refractory to 'standard' therapies, neurosurgical procedures may be considered. Guidelines for safe and ethical conduct of such procedures have previously and independently been proposed by various local and regional expert groups.

Methods To expand on these earlier documents, representative members of continental and international psychiatric and neurosurgical societies, joined efforts to further elaborate and adopt a pragmatic worldwide set of guidelines. These are intended to address a broad range of neuropsychiatric disorders, brain targets and neurosurgical techniques, taking into account cultural and social heterogeneities of healthcare environments.

Findings The proposed consensus document highlights that, while stereotactic ablative procedures such as cingulotomy and capsulotomy for depression and obsessive-compulsive disorder are considered 'established' in some countries, they still lack level I evidence. Further, it is noted that deep brain stimulation in any brain target hitherto tried, and for any psychiatric or behavioural disorder, still remains at an investigational stage. Researchers are encouraged to design randomised controlled trials, based on scientific and data-driven rationales for disease and brain target selection. Experienced multidisciplinary teams are a mandatory requirement for the safe and ethical conduct of any psychiatric neurosurgery, ensuring documented refractoriness of patients, proper consent procedures that respect patient's capacity and autonomy, multifaceted preoperative as well as postoperative long-term followup evaluation, and reporting of effects and side effects for all patients.

Interpretation This consensus document on ethical and scientific conduct of psychiatric surgery worldwide is designed to enhance patient safety.

\section{BACKGROUND}

The majority of patients affected by psychiatric disorders can be managed effectively by means of pharmacological therapies, psychotherapy and in some cases, more technical interventions such as electroconvulsive therapy. These evidence-based treatments may be used either alone or in combination. However, a substantial minority of patients either does not respond, fails to sustain response or experiences unacceptable adverse effects. It is for these patients, who are even more at risk with nontreatment, that the use of neurosurgical procedures such as stereotactic focal ablative surgery or deep brain stimulation (DBS) may be considered. ${ }^{12}$ Case reports, case series and small-scale clinical trials of neurosurgical interventions have been reported in patients with obsessive-compulsive disorder (OCD), major depressive disorder (MDD), substance abuse/ addiction and anorexia nervosa, among others.

The published experience of DBS for these conditions would appear to have intuitive appeal since DBS is both adjustable and in most cases reversible in contrast to stereotactic ablative techniques. DBS nonetheless requires an invasive implantation of a permanent device in the brain, with the inherent risks of the surgical procedure and the burden of managing, maintaining and replacing the device. Until scientifically proven otherwise, DBS is not superior to ablative surgery for psychiatric disorders. Clinical studies in this field may provide an unprecedented opportunity for fundamental work with regard to examining disease pathophysiology and the mechanisms of action of these therapies. ${ }^{3}$

\section{METHODS}

The Committee for Neurosurgery for Psychiatric Disorders, as part of the World Society for Stereotactic and Functional Neurosurgery (WSSFN) and the European Society for Stereotactic and Functional Neurosurgery (ESSFN), partnering with the Working Group 'Deep Brain Stimulation in Psychiatry: Guidance for Responsible Research and Application', along with the Psychiatric Neurosurgery Committee of the American Society for Stereotactic and Functional Neurosurgery (ASSFN), the Latin American Society for Stereotactic and Functional Neurosurgery (SLANFE), the 
Asian-Australasian Society for Stereotactic and Functional Neurosurgery (AASSFN) and the World Psychiatric Association (WPA), propose an expanded set of guidelines to articulate a consensus summary of clinical research standards that are applicable to testing of DBS and ablative neurosurgery in addition to other emerging neurosurgical interventions for neuropsychiatric disorders.

The need for such consensus of guidelines was first identified by the Committee for Neurosurgery for Psychiatric Disorders (WSSFN) in early 2011. Then the first text was drafted and revised by $\mathrm{HW}$ and $\mathrm{BN}$, respectively, based on literature review. MH provided an important part of the references. This text was presented by BN to the Working Group 'Deep Brain Stimulation in Psychiatry: Guidance for Responsible Research and Application' for further in-depth discussion, leading to a very extensively modified version of the previous text. Afterwards, it was distributed internally by $\mathrm{BN}$ among representatives of different international societies (WSSFN, ESSFN, ASSFN, SLANFE, AASSFN and WPA), from whom remarks were received, leading to an optimised consensus text, which was finally approved and endorsed by the representatives of the different societies in late 2011/early 2012.

The guidelines for neurosurgery for psychiatric disorders presented in this document recognise the Declaration of Helsinki, issued by the World Medical Association in 1964, amended several times, as the fundamental document in the field of ethics in biomedical research.

The following guidelines are built directly upon the core elements of previously published guidelines, reviews, correspondences and legislation from expert neurosurgical, neurological, psychiatric and neuroethics groups, and health administrations around the world with an interest in the practice of neurosurgery for psychiatric disorders. We have adopted a pragmatic view in defining a set of guidelines that attempt to serve the range of neuropsychiatric disorders and, crucially, the cultural and religious diversity and heterogeneity of healthcare environments of the international collaborative partners engaged in these endeavours. The guidelines represent a shared attempt to articulate these norms at this time. We appreciate that views can and will evolve over time; therefore, we encourage and welcome the start of an iterative process. The consensus group wishes to emphasise the potential importance of neurosurgical interventions in the future management of psychiatric disorders. These guidelines are not meant to inhibit, but rather to guide ethical and effective research in order to facilitate proper development of promising therapies. They represent an international multidisciplinary consensus on best ethical practices, norms and professional behaviours both in clinical and research settings.

The scope of neurosurgical interventions

for psychiatric disorders

Neurosurgical therapies for psychiatric disorders range from those that have been in routine use in specialist centres for several decades (eg, anterior cingulotomy for MDD, anterior capsulotomy for OCD), to those that remain highly experimental and have only been tested in very small number of patients (eg, DBS for anorexia nervosa). However, despite the lengthy history and the weight of publications, associated with lesion procedures in particular, the accumulated evidence supporting the application of all neurosurgical treatments for psychiatric disorders requires to be strengthened. While certain procedures are considered to represent 'established' practice for severe, treatment-refractory psychiatric disorders in some countries (eg, radiofrequency anterior capsulotomy for severe, treatment-refractory OCD in Belgium, thermal anterior cingulotomy for MDD and OCD in the USA, Scotland, South Korea and elsewhere), the nature of these and many other procedures in neurosurgery, including DBS for psychiatric disorders, remains at a 'proof-of-principle' investigational stage of development. ${ }^{4}$

Current practised stereotactic ablative procedures do not have level I evidence with randomised controlled trials, but their safety and efficacy are supported by level II evidence in treatment-refractory MDD and OCD. However, this degree of evidence is not yet available for 'new' lesioning methods such as gamma knife and stereotactic-focused ultrasound.

In this delicate field of neurosurgery for psychiatric disorders, it seems reasonable to state the following requirement before the surgical intervention can be stated as 'approved therapy'. At least two blinded (if possible) randomised controlled clinical trials from two different groups of researchers need to be published, both showing an acceptable risk-benefit ratio, at least comparable with other existing therapies.

Furthermore, there is a resurgence of ablative procedures in resource-poor contexts, where access to medication, psychotherapy and more expensive neurosurgical interventions like DBS is limited. Ablative surgery also becomes an alternative in cases where a DBS procedure has failed to control the patient's symptoms or where DBS or other neuromodulatory strategies are inappropriate or impractical.

We encourage researchers to design independent, randomised and blinded (where possible) controlled trials, with the least possible conflict of interest and bias, to strive towards the generation of level I (U.S. Preventive Services Task Force) or level A (U.K. National Institute of Clinical Excellence (NICE) and the Scottish Intercollegiate Guidelines Network (SIGN)) clinical evidence with regard to neurosurgical procedures for psychiatric disorders. Unfortunately, different organisations use different systems to grade evidence and recommendations. Therefore, a new system-called GRADE-is gaining more acceptance internationally to establish the quality of evidence and the strength of recommendation. ${ }^{5}$ Robust, data-driven, evidence-based rationales for disease and brain target selection are required. They will provide further protection of patients' safety, improve clinical choice and outcomes, and provide the basis for studies of disease mechanisms. ${ }^{3}$ Further, prior to proceeding to selection of a new brain target or disease, consultation with peers in the field is strongly recommended. High-quality pilot studies, honestly reported with details and accuracy, have usually allowed the generation of new discoveries and can pave the way for bigger clinical trials. The point here is not to blame controlled trials, but to underline that sometimes small pilot studies can be used to guide and prepare larger, international clinical trials by providing preliminary data that will avoid fishing expedition (eg, for the choice of targets and parameters of stimulation).

\section{Involvement of ethics committees and institutional review boards}

An independent Ethics Committee or Institutional Review Board (IRB) must have ethical and regulatory oversight for all investigational neurosurgery for psychiatric disorders. These committees, in tandem with local and national regulatory agencies, such as the Food and Drug Administration (FDA) in the USA, the European Medicines Agency (EMA) in the European Union and their counterparts worldwide, must review and oversee all aspects of the investigational protocol. Special attention must be paid to the process of informed consent, avoidance of therapeutic misconception, proportionality in research, the assessment of investigative teams, as well as independent experts 
for the interdisciplinary composition necessary to conduct this work. ${ }^{6}$ Particular care is required in cases involving vulnerable populations (eg, children, those in hierarchical relationships such as military members, students and prisoners) and in cases of surrogate decision making when one makes decisions for others. ${ }^{7} 8$ Ethical norms that govern these decisions should reflect the patient/subject's prior wishes, if they are known, and be in their best interest. For complex cases, functional neurosurgeons, together with members of the psychiatric team, should seek expert bioethics consultation.

A critical distinction must be made for all psychiatric neurosurgery, be it ablative or DBS, whether the intervention has reached therapeutic status or remains investigational. Regulation of the former should be handled as clinical practice and the latter as research, requiring discrete oversight, including a Data Safety Monitoring Board (DSMB) when indicated. Investigators should be careful not to prematurely designate an investigational intervention as the standard of care, based on historic precedent or on limited data, and should seek the advice and guidance of ethics bodies to avoid idiosyncratic practices.

\section{Preoperative evaluation and patient selection criteria}

All candidates for neurosurgery for psychiatric disorders should meet generally accepted clinical criteria for severity, chronicity, disability and treatment refractoriness. ${ }^{9}$ A comprehensive preoperative assessment by independent experts in the management of psychiatric disorders ensures that all candidates meet rigorous inclusion and exclusion criteria. ${ }^{10}$ Although asking advice from independent experts is not common practice in medicine and is difficult to install as a mandatory process worldwide, it has proven to be of value. ${ }^{11}$ Evaluations should use standardised rating scales, ${ }^{10}$ including scales rating disability and quality of life. The definition of treatment refractoriness will vary by disorder. ${ }^{4}$ The suicide risk should be taken into account in all individuals participating in neurosurgery for psychiatric disorders before surgery. ${ }^{4}$ All patients should complete a preoperative neuropsychological assessment that includes an evaluation of the patient's current cognitive abilities, psychiatric status, personality and interpersonal functioning, goals and expectations of surgery, treatment adherence and level of family or other psychosocial support. $^{12}$

There should be documentation of failure (eg, due to disabling side effects or lack of efficacy) or limited response to trials of available standard therapies with adequate dose and duration (eg, pharmacotherapy, behavioural or cognitive therapy, electroconvulsive therapy). ${ }^{13}{ }^{14}$ There should be no other reasonable, evidence-based and less intrusive alternatives available, taking into account the aggregate risks, benefits and side effects of the proposed intervention. ${ }^{13}$ Furthermore, there should be little hope for spontaneous recovery and a potential for meaningful recovery after surgery. ${ }^{13}$

Treating the patient remains the primary aim of clinical research. Indeed, the fact that, for example, DBS is in general reversible and opens windows to the brain should not be used first for answering an exciting physio-pathological question, while using the clinical aim only as a pretext.

Decision-making capacity, autonomy and informed consent Informed consent must be obtained from competent patients. This requires an explanation of the risks, benefits and alternatives, as well as a context of the individuals' free choice. Risks are not only limited to known surgical risks but can include unknown risks associated with stimulation, ablation or other forms of modulation at novel sites. The risks of treatment must also be placed in a clinical context and balanced against the risks of no treatment. The consent process should include discussion of what is and is not known of the long-term consequences of neurosurgery for psychiatric disorders. ${ }^{4}$ It should be explained clearly that neurosurgery for psychiatric disorders is only one aspect of a comprehensive treatment programme that should continue after surgery. ${ }^{4}$ The patient should understand that neurosurgery for psychiatric disorders aims for a symptomatic treatment of psychiatric impairments, but may not be able to 'cure' the disease process. ${ }^{1}$

A. An assessment of the patients' decision-making capacity to consent should be carried out for each potential subject in early phase studies of neurosurgery for psychiatric disorders. ${ }^{4}$ The methods used should take into account the potential confounds of psychiatric symptoms. ${ }^{4}$ Desperation can lead to decisions in a hurry, in favour of surgery. ${ }^{15}$ Decisional capacity may change over the course of illness or treatment, like in depression, and therefore should be regularly assessed. ${ }^{15}$ For patients to have decisional capacity, they must satisfy the following three criteria:

- Sufficient comprehension of the importance of the protected personal spheres (physical and mental) into which a neurosurgical intervention intrudes and of the scope and risk of the intervening clinical measures.

- Sufficient judgement, that is, the ability to assess the consequences of the intervention in light of one's own matters and interests.

- Sufficient ability to take self-governed decisions, that is, the basic capability to decide and act according to one's own insights and judgements.

B. The provision of 'care' to competent individuals, with the capacity to consent, without informed consent, is a violation of ethical norms and disrespectful of personhood.

C. It is acceptable to obtain surrogate consent when the individual lacks decision-making capacity. Such use of surrogate decision-makers should represent extremely rare cases. It requires special vigilance as surrogates may, on purpose or unknowingly, pursue their own interests at the expense of the patient. ${ }^{16}$ Local legislation may govern such clinical situations in different countries. ${ }^{17}{ }^{18}$ In general, patients who cannot give their own free and informed consent should not be considered candidates for psychiatric neurosurgery unless there is a legally authorised representative and specific laws governing such situations.

An example where a surrogate decision-maker may intervene could be a person with extremely low IQ with extreme autoaggression. There are known cases who perform laparotomy on themselves or pull out one eye and the second eye is in danger. If no other therapy would help, one may think of a neurosurgical procedure that decreases the likelihood of extreme autoaggression. But even in this life-threatening case a surrogate decision-maker only comes into play when every effort has been made to obtain a positive consent from the patient.

D. Patient consent should be maintained and monitored throughout the neurosurgery for psychiatric disorders process, and patients must be free to halt their participation voluntarily. ${ }^{9}$

\section{Experienced multidisciplinary team}

Neurosurgery for psychiatric disorders should not be decided by, nor performed by, an individual in isolation and acting alone regardless of specialty. These procedures require an expert multidisciplinary team that includes trained stereotactic and functional neurosurgeons, working in a team with psychiatrists, 
neurologists and neuropsychologists. The team should be specialised in the various target disorders and be able to provide comprehensive care. ${ }^{19}$ Neurosurgeons should use modern, current standard techniques such as MRI and computerised stereotactic planning software. It is a neurosurgeons' important responsibility to check and maintain accuracy and reliability of the stereotactic system. Postoperative imaging is mandatory (eg, for documentation of the electrode position or place and extent of the lesion).

The composition of the team should be adjusted to the disorder and may involve a neuroethicist, depending on the idiosyncratic demands of the work. ${ }^{6}$ Ancillary specialists may also be incorporated into these teams to provide expertise in social work, rehabilitation, psychotherapy and vocational training. For best protection of public and profession, members of multidisciplinary neurosurgical groups should monitor their colleagues to ensure that all members adhere to the proposed guidelines.

It is mandatory to reach a complete consensus with regard to patient selection, preoperative evaluation and neurosurgical therapy among neurosurgeons, psychiatrists and other members of the team as a sine qua non condition. In case of disagreement, any member of the team should not act alone, and outside expert evaluation should be sought.

\section{Legitimate therapeutic indications}

We agree with the landmark 1977 US National Commission Report on Psychosurgery: "The Commission affirms that the use of psychosurgery for any purpose other than to provide treatment to individual patients would be inappropriate and should be prohibited. (Italics in original) Accordingly, the Commission is recommending safeguards that should prevent the performance of psychosurgery for purposes of social or institutional control or other such misuse."2021

Neurosurgery for psychiatric disorders should never be performed for political, law enforcement or social purposes, but with therapeutic intent aimed at the restoration of normal function and amelioration of distress and suffering. 9 13

These patients may come from a challenging socioeconomic background. However, they should not be deprived of, nor given, a lesser opportunity to participate in cutting-edge research that may have an important impact in the treatment of their condition. This research should be available to all patients irrespective of race, ethnicity, gender, class, religion, sexual orientation or any other potential cause for bias.

\section{Conflicts of interest}

The potential for ethical conflicts of interest exists because this work is often reliant upon collaborations between academia, industry and the clinic. ${ }^{3}$ Even though device-related or biotherapeutic companies may economically support a clinical study to benefit the interests of patients, it is undeniable that a legitimate commercial interest in making a profit coexists. In consequence, there is a potential risk that this aspect may hamper the transparency of the study. ${ }^{22}$

Patients and/or their legally authorised representatives should be made fully aware of the potential conflicts of interest in all informed consent discussions. ${ }^{9}$ Investigators should be transparent with disclosures of financial conflicts of interests, including (but not limited to) corporate relationships, consulting fees, honoraria, research funding and intellectual property rights. This information should be shared with prospective participants or their surrogates, colleagues, institutional officials and regulators as required by local laws and professional norms. Investigators with potential conflicts of interest should not be precluded categorically from doing research if the conflict is properly and impartially managed. ${ }^{3}$

\section{Postoperative evaluation and long-term follow-up}

The ethical principle of non-abandonment obliges clinicians to follow all patients/subjects longitudinally or until proper transfer of care to a qualified clinician occurs. This provision of ongoing care is especially important because specialty care is not routinely available in the community. ${ }^{21} 23$

A. All patients initially enrolled in any treatment programme or clinical trial for neurosurgery for psychiatric disorders should complete comprehensive postoperative assessments, including neurological, psychiatric and neuropsychological evaluations, and should be followed up regularly. ${ }^{10} 2425$

B. Clinical research teams should report on the outcome for all patients, including failed or withdrawn cases, as is mandatory in any scientific report. ${ }^{24}$ Patient trust to enrol in such trials requires that all efforts be made to collect as many scientific data as reasonably possible to not only determine safety and clinical efficacy, but to understand the 'therapy' and the reason for the resulting outcomes. This includes the use of multiple clinical ratings and objective scientific measures, such as functional imaging, wherever possible.

C. In addition to disease-specific symptom outcomes, outcomes in domains such as activities of daily living, cognition, quality of life and global improvement (including family and patient perception) should be considered. ${ }^{4}$ Social adjustment following neurosurgery may be challenging for many patients. ${ }^{1}$

D. Research and clinical protocols should include support for long-term safety and efficacy studies on neurosurgery for psychiatric disorders for at least 5-10 years of follow-up. ${ }^{4}$ Regulatory agencies should require that device manufacturers collect long-term follow-up data on safety and efficacy. ${ }^{4}$

E. It should not be considered inherently problematic that neurosurgical interventions have the potential to cause personality changes. In view of the fact that many psychiatric disorders may bring about undesirable changes of a patient's personality, it can even be the intended outcome of such interventions to modify personality by undoing the pathological changes. ${ }^{26}$ However, all psychiatric and nonpsychiatric side effects should be documented. ${ }^{27}$

F. An independent registry, at this moment not yet available, should ideally include de-identified data on all individuals undergoing neurosurgery for psychiatric disorders. ${ }^{4} 28$

\section{Author affiliations}

${ }^{1}$ Research Group of Experimental Neurosurgery and Neuroanatomy, Department of Neurosciences, KU Leuven, Leuven, Belgium

${ }^{2}$ Chair of Committee of Neurosurgery for Psychiatric Disorders of the WSSFN and Department of Neurosurgery, UZ Leuven, Leuven, Belgium

${ }^{3}$ Working Group 'Deep Brain Stimulation in Psychiatry: Guidance for Responsible Research and Application'. This Working Group is organised by the Europäische Akademie GmbH (Bad Neuenahr-Ahrweiler, Germany, http://www.ea-aw.de). It consists of an interdisciplinary and international team of neurosurgeons, neurologists, psychiatrists, neuropsychologists, bioethicists, philosophers and legal scholars, analysing ethical issues arising from the application of Deep Brain Stimulation for Psychiatric Disorders. Questions such as critical issues around regulatory processes and ethical guidance for the management of conflicts of interest for researchers, engineers and clinicians engaged in the development of therapeutic deep brain stimulation have been comprehensively studied and the results have been published under common authorship.

${ }^{4}$ WSSFN Committee on Neurosurgery for Psychiatric Disorders

${ }^{5}$ Department of Psychiatry, Emory University School of Medicine, Atlanta, GA, USA ${ }^{6}$ Department of Clinical Neuroscience, UCL Institute of Neurology, Queen Square, London, Umea University, Umea, Sweden 
${ }^{7}$ Chair of the Committee of Neurosurgery for Psychiatric Disorders, Belgium and Belgium and Department of Psychiatry, UZ Leuven, Leuven, Belgium

${ }^{8}$ Deutsches Referenzzentrum für Ethik in den Biowissenschaften, Bonn, Germany ${ }^{9}$ Universität Hamburg/Juristische Fakultät Lehrstuhl für Strafrecht und Rechtsphilosophie, Hamburg, Germany

${ }^{10}$ Center for Neurological Restoration, Cleveland Clinic, Cleveland, OH, USA ${ }^{11}$ Department of Neurosciences, Institute of Neurology of Goiânia, Stereotactic and Functional Neurosurgery Service, Medical School, Federal University of Goiás, Medical School, Pontifical Catholic University of Goiás, Goiânia, Brazil

${ }^{12}$ Advanced Interventions Service and Division of Neuroscience, University of Dundee, Ninewells Hospital and Medical School, Dundee, Scotland, UK

${ }^{13}$ Past-president of WSSFN and Chair of Committee of Stereotactic and Functional Neurosurgery, World Federation of Neurosurgical Societies (WFNS), and Department of Neurosurgery, World Federation of Neurosurgical Societies (WFNS), Tokyo Women's Medical University, Tokyo, Japan

${ }^{14}$ Past-president of WSSFN and Department of Neurosurgery, Toronto Western Hospital, Toronto, Canada

${ }^{15}$ Department of Neurosurgery and Clinical Neuroscience, Karolinska Institutet and University Hospital, Stockholm, Sweden

${ }^{16}$ Stereotactic and Functional Neurosurgery Program, Jaslok Hospital and Research Centre, Mumbai, India

${ }^{17}$ Department of Neurosurgery, Instituto Neurologico C. Besta, Milano, Italy

${ }^{18}$ Department of Functional Neurosurgery, Hôpital La Timone, Aix-Marseille Université, Marseille, France

${ }^{19}$ Department of Neurosciences, King Faisal Specialist Hospital and Research Centre, AlFaisal University, Riyadh, Saudi Arabia

${ }^{20}$ Center for Functional Neurosurgery, Shanghai Jiao Tong University Rui Jin Hospital, Shanghai, China

${ }^{21}$ Centre of Neurosciences Department of Neurosurgery, Ninewells Hospital and Medical School Dundee, Dundee, Scotland, UK

${ }^{22}$ Department of Neurosurgery, Hofstra North Shore LIJ School of Medicine, North Shore University Hospital, Manhasset, NY, USA

${ }^{23}$ Member of the Psychiatric Surgery Committee of the ASSFN and Department of Neurological Surgery, Weill Cornell Medical College, NY, USA

${ }^{24}$ Member of the Psychiatric Surgery Committee of the ASSFN and Department of Neurosurgery, Massachusetts General Hospital, Harvard Medical School, Boston,

MA, USA

${ }^{25}$ Past President, American Society of Stereotactic and Functional Neurosurgery

(ASSFN), Department of Neurosurgery, American Society of Stereotactic and

Functional Neurosurgery (ASSFN), Ohio State University, Ohio, USA

${ }^{26}$ President of ESSFN and WSSFN, Department of Neurosurgery, Medical University of Hannover, Hannover, Germany

${ }^{27} \mathrm{KU}$ Leuven, Belgium

${ }^{28}$ Department of Neurosurgery, Academic Medical Center, Amsterdam, The Netherlands

${ }^{29}$ President of WPA, Department of Psychiatry and Behavioral Sciences, Miami Miller School of Medicine, Miami, USA

${ }^{30}$ President of Asian Australasian Society for Stereotactic \& Functional Neurosurgery Department of Neurosurgery, Yonsei University College of Medicine, Seoul, Korea

${ }^{31}$ Committee of Neurosurgery for Psychiatric Disorders, Belgium, Kortenberg, Belgium

${ }^{32}$ Department of Neurosurgery, Toronto Western Hospital, Toronto, Canada

${ }^{33}$ Department of Stereotactic Neurosurgery, Otto-von-Guericke University Magdeburg and Leibniz-Institute for Neurobiology, Magdeburg, Germany

${ }^{34}$ Department of Neurosurgery, Brown University, Providence, RI, USA

${ }^{35}$ Department of Neurosurgery, Xuanwu Hospital, Beijing, China

${ }^{36}$ Chair of the Focus Group 'Deep Brain Stimulation in Psychiatry: Guidance for Responsible Research and Application', Chair of the Task Force on Brain Stimulation of the World Federations of Societies of Biological Psychiatry, Chair of the Section of Experimental Brain Stimulation Methods of the German Association of Psychiatry, Psychotherapy and Psychosomatics and member of the Operational Committee on Sections of the World Psychiatric Association. He is professor of Psychiatry and Psychotherapy at the University of Bonn, Germany, and Associate Professor of Psychiatry and Mental Health at The Johns Hopkins University, Baltimore, MD, USA.

Acknowledgements Joseph J Fins, M.D., M.A.C.P., Chief of the Division of Medical Ethics of Weill Medical College of Cornell University and member of the Europäische Akademie's Working Group 'Deep Brain Stimulation in Psychiatry: Guidance for Responsible Research and Application' made co-first author contributions to this paper and agreed with its conclusions. Professor Fins has declined to be listed as an author. We thank Damianos Sakas, Jairo Espinoza, Marcos Baabor, Fabián Piedimonte, Terry Coyne and Takamitsu Yamamoto for their critical comments and final approval of the manuscript.

Contributors BN initiated the study, contributed to the writing and reviewing of the manuscript. He coordinated the interactions between different international societies and authors, contacted relevant persons, and finalised the manuscript. HW summarised past literature and drafted the manuscript. He also participated in revising the manuscript and together with BN shares the co-first-authorship. HM contributed to concept of paper, writing of early draft and editing of final draft. $\mathrm{MH}$ contributed to literature search, study design, data collection and analysis, writing parts of manuscript and discussion of intellectual content. LG participated in discussions and meetings for development of the current guidelines and tested them for support in the psychiatric community. She critically revised the manuscript and commented on it at several stages. TG did extensive editing of the first draft of the article and gave critical comments on the final manuscript. RM contributed substantially in arguments and wording to sections 'Decision-making capacity, autonomy and informed consent' and 'Legitimate therapeutic indications' and edited work on the entire text. CK did modification and significant editing and revision of original draft. OV-F, as the Past-Chairman of the Committee for Neurosurgery for Psychiatric Disorders of the WSSFN, started the process of bringing together neurosurgeons within the WSSFN to talk about ethical aspects of neurosurgery for psychiatric disorders. OV-F helped in the study design, data interpretation and review of this manuscript. KM, TT, AML, AA, MS, RS, JWC, NL, JV, RC and YL reviewed and agreed with the manuscript. GS contributed to writing and literature search. PD helped in conceiving the idea and implementation as a task force member. He has provided inputs about the guideline format and has also reviewed and edited the manuscript. Thus he has been involved in the study design, interpretation of the manuscript and its final writing. GB contributed in literature search and study design. JR did extensive reviewing of the manuscript. BS did literature search and study design and participated in conferences to discuss the guideline. SE discussed and participated in the evaluation and agreed with the layout. He proofread the manuscript and critically reviewed it. MK contributed to writing and editing the manuscript. EE contributed to the content and reviewed the manuscript. AR performed literature search, writing and editing of the manuscript. JKK participated in discussion of contents and review of the manuscript. PH critically revised the manuscript and commented on it. PR is a reviewer of the manuscript. PC contributed as chairman of the Belgian Committee of Neurosurgery for Psychiatric Disorders. TS drafted the manuscript together with the first authors, repeatedly edited as the manuscript progressed and approved the final manuscript.

Funding The Working Group 'Deep Brain Stimulation in Psychiatry: Guidance for Responsible Research and Application' was funded by the Volkswagen Foundation (Hanover, Germany).

Competing interests $\mathrm{BN}$ received grants for research, education and travel from Medtronic. He owns a patent on DBS for OCD. He has a chair, 'stereotactic neurosurgery for psychiatric disorders', a donation from Medtronic. HW is a recipient of the IWT Baekeland Mandate (industrial partner: Synaptix). HM is Consultant of St. Jude Medical/Neuromodulation Inc. Licensing of IP: SJM/Neuromodulation Inc. MH has received honoraria and travel expense from Medtronic and St. Jude for speaking at meetings. LG has a chair, 'stereotactic neurosurgery for psychiatric disorders', a donation from Medtronic. OV-F has occasionally received travel grants from the representatives of Medtronic, St. Jude and Elekta in Brazil. KM has chaired advisory boards for studies of deep brain stimulation for obsessive-compulsive disorder sponsored by Medtronic. He has received educational grants from Cyberonics Inc \& Schering Plough and received research project funding from Merck Serono \& Reckitt Benckiser and also from St. Jude Medical for a multicentre clinical trial of deep brain stimulation for depression. He has received travel and accommodation support to attend meetings from Medtronic and St. Jude Medical. TT received consultancy from St. Jude Japan; grants/grants pending and speaking fees from Daiichi-Sankyo Pharmaceutical Company. AML is a consultant of Medtronic, St. Jude, Boston Scientific, Codman and Functional Neuroscience Inc. AR received neurological research support from Medtronic. JKK is a consultant to Medtronic. JV receives consulting fees from Medtronic and Sapiens and occasionally speaking fees from Medtronic and St. Jude Medical. TS receives unrestricted funding for DBS for depression from Medtronic Inc., a manufacturer of DBS devices. He obtained a grant from the Volkswagen Foundation to establish a focus ground on neuroethical issues regarding DBS's application for neuropsychiatric disorders. TG, $R M, C K, G S, P D, G B, J R, A A, B S, S E, M S, M K, E E, P H, R S, P R$, JWC, PC, NL, RC and $Y L$ declare that there are no conflicts of interest.

Provenance and peer review Not commissioned; externally peer reviewed.

Open Access This is an Open Access article distributed in accordance with the Creative Commons Attribution Non Commercial (CC BY-NC 3.0) license, which permits others to distribute, remix, adapt, build upon this work non-commercially, and license their derivative works on different terms, provided the original work is properly cited and the use is non-commercial. See: http://creativecommons.org/ licenses/by-nc/3.0/

\section{REFERENCES}

1 Clausen J. Ethical brain stimulation: neuroethics of deep brain stimulation in research and clinical practice. Eur J Neurosci 2010;32:1152-62.

2 Pressman J. Last resort: psychosurgery and the limits of medicine. Cambridge: Cambridge University Press, 2002 
3 Fins JJ, Schlaepfer TE, Nuttin B, et al. Ethical guidance for the management of conflicts of interest for researchers, engineers and clinicians engaged in the development of therapeutic deep brain stimulation. J Neural Eng 2011;8: 033001

4 Rabins P, Appleby BS, Brandt J, et al. Scientific and ethical issues related to deep brain stimulation for disorders of mood, behavior, and thought. Arch Gen Psychiatry 2009;66:931-7.

5 Guyatt GH, Oxman AD, Schunemann HJ, et al. GRADE guidelines: a new series of articles in the Journal of Clinical Epidemiology. J Clin Epidemiol 2011;64:380-2.

6 Fins JJ, Rezai AR, Greenberg BD. Psychosurgery: avoiding an ethical redux while advancing a therapeutic future. Neurosurgery 2006;59:713-16.

7 Buchanan AE, Brock DW. Deciding for others: the ethics of surrogate decision making. Cambridge: Cambridge University Press, 1990.

8 Fins JJ. A proposed ethical framework for interventional cognitive neuroscience: a consideration of deep brain stimulation in impaired consciousness. Neurol Res 2000:22:273-8.

9 Nuttin B, Gybels J, Cosyns P, et al. Deep brain stimulation for psychiatric disorders. Neurosurgery 2002;51:519.

10 Mink JW, Walkup J, Frey KA, et al. Patient selection and assessment recommendations for deep brain stimulation in Tourette syndrome. Mov Disord 2006;21:1831-8.

11 Gabriëls L, Nuttin B, Cosyns P. Applicants for stereotactic neurosurgery for psychiatric disorders: role of the Flemish advisory board. Acta Psychiatr Scand 2008;117:381-9.

12 Ford PJ, Kubu CS. Stimulating debate: ethics in a multidisciplinary functional neurosurgery committee. J Med Ethics 2006:32:106-9.

13 Kuhn J, Gaebel W, Klosterkoetter J, et al. Deep brain stimulation as a new therapeutic approach in therapy-resistant mental disorders: ethical aspects of investigational treatment. Eur Arch Psychiatry Clin Neurosci 2009;259(Suppl 2):S135-41.

14 Visser-Vandewalle V, Ackermans $L$, van der Linden $C$, et al. Deep brain stimulation in Gilles de la Tourette's syndrome. Neurosurgery 2006;58:E590.

15 Dunn LB, Holtzheimer PE, Hoop JG, et al. Ethical issues in deep brain stimulation research for treatment-resistant depression: focus on risk and consent. $A J O B$ Neurosci 2011;2:29-36.
16 Kim SY. The ethics of informed consent in Alzheimer disease research. Nat Rev Neurol 2011;7:410-14.

17 Ministry of Health of the People's Republic of China. Notification from Ministry of Health: regarding strengthening management and related issues in neurosurgery for psychiatric disorders. [in Chinese]. http://www.moh.gov.cn/mohbgt/pw10806/ 200804/33288.shtml (accessed 13 Aug 2013).

18 Wu H, Gabriëls L, Nuttin B. Neurosurgery for psychiatric disorders in the people's republic of China-responsibilities of international societies. AJOB Neurosci 2012;3:56-9.

19 Schlaepfer TE, George MS, Mayberg H. WFSBP guidelines on brain stimulation treatments in psychiatry. World J Biol Psychiatry 2010:11:2-18.

20 The National Commission for the Protection of Human Subjects of Biomedical and Behavioral Research. Use of psychosurgery in practice and research: report and recommendations of national commission for the protection of human subjects of biomedical and behavioral research. Federal Register 1977;42:26318-32.

21 Fins JJ. From psychosurgery to neuromodulation and palliation: history's lessons for the ethical conduct and regulation of neuropsychiatric research. Neurosurg Clin N Am 2003; 14:303-19, ix-x.

22 Schermer M. Ethical issues in deep brain stimulation. Front Integr Neurosc 2011:5:17.

23 Fins JJ. Deep brain stimulation, deontology and duty: the moral obligation of non-abandonment at the neural interface. J Neural Eng 2009;6:050201.

24 Hariz MI. Psychosurgery, deep brain stimulation, and the re-writing of history Neurosurgery 2008;63:E820; author reply E.

25 Lipsman N, Bernstein M, Lozano AM. Criteria for the ethical conduct of psychiatric neurosurgery clinical trials. Neurosurg Focus 2010;29:E9.

26 Synofzik M, Schlaepfer TE. Electrodes in the brain: ethical criteria for research and treatment with deep brain stimulation for neuropsychiatric disorders. Brain Stimul 2011;4:7-16.

27 Bell E, Mathieu G, Racine E. Preparing the ethical future of deep brain stimulation. Surg Neurol 2009;72:577-86; discussion 86.

28 Synofzik M, Fins JJ, Schlaepfer TE. A neuromodulation experience registry for deep brain stimulation studies in psychiatric research: rationale and recommendations for implementation. Brain Stimul 2012;5:653-5. 